\title{
RECENT ADVANCES IN QUANTUM MONTE CARLO METHODS
}




\title{
Recent Advances in Computational Chemistry
}

\author{
Editor-In-Charge \\ Delano P. Chong, Department of Chemistry, University of British Columbia, \\ Canada
}

\author{
Published \\ Recent Advances in Density Functional Methods, Part I \\ ed. D. P. Chong \\ Forthcoming \\ Recent Advances in Density Functional Methods, Part II \\ ed. D. P. Chong \\ Recent Advances in Coupled-Cluster Methods \\ ed. R. J. Bartlett
}




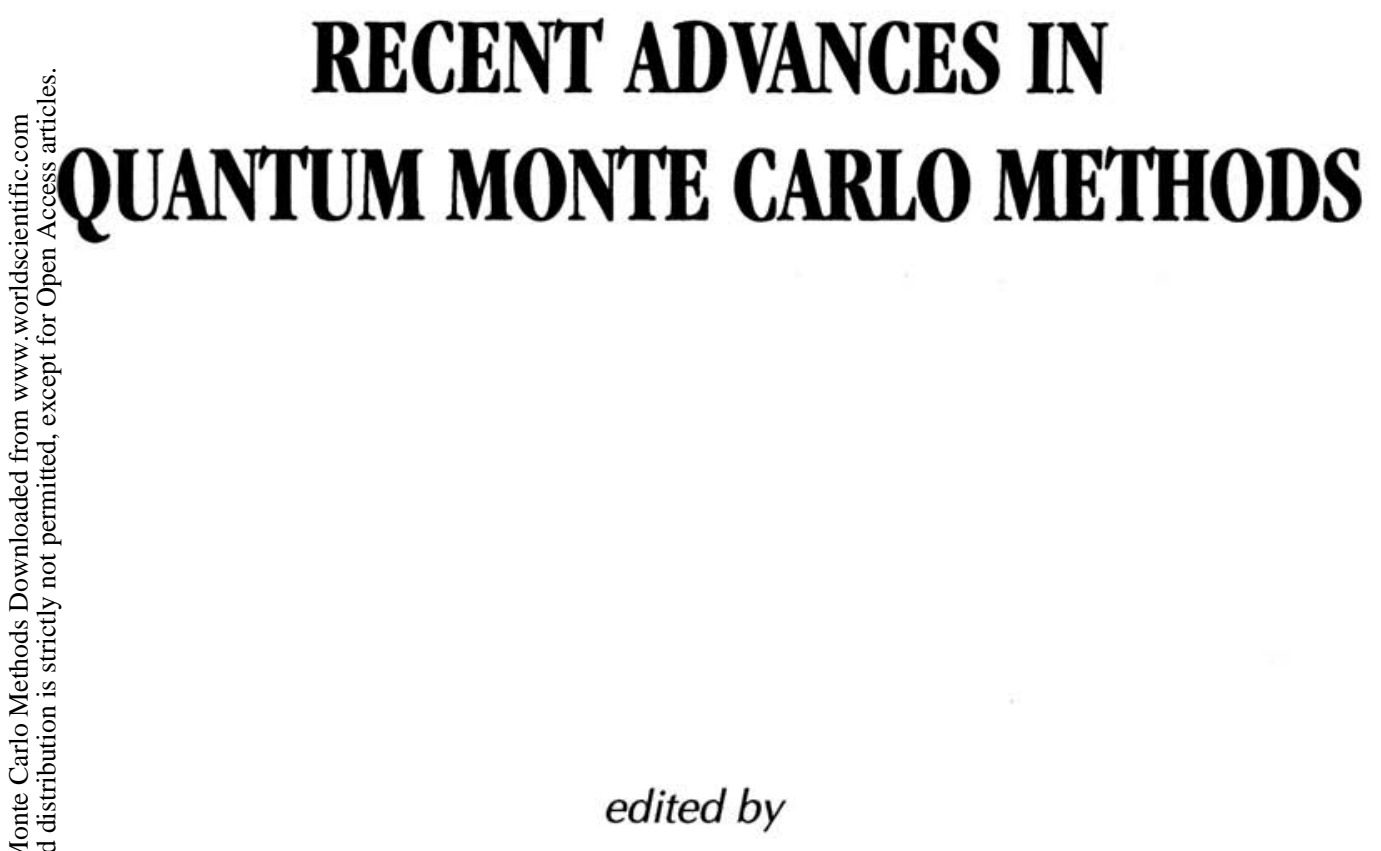

\section{William A. Lester Jr.}

University of California

Berkeley, USA 


\section{Published by}

World Scientific Publishing Co. Pte. Ltd.

P O Box 128, Farrer Road, Singapore 912805

USA office: Suite 1B, 1060 Main Street, River Edge, NJ 07661

UK office: 57 Shelton Street, Covent Garden, London WC2H 9HE

\section{British Library Cataloguing-in-Publication Data}

A catalogue record for this book is available from the British Library.

\section{RECENT ADVANCES IN QUANTUM MONTE CARLO METHODS \\ Copyright @ 1997 by World Scientific Publishing Co. Pte. Ltd. \\ All rights reserved. This book, or parts thereof, may not be reproduced in any formor by any means, electronic or mechanical, including photocopying, recording or any information storage and retrieval system now known or to be invented, without written permission from the Publisher.}

For photocopying of material in this volume, please pay a copying fee through the Copyright Clearance Center, Inc., 222 Rosewood Drive, Danvers, MA 01923, USA. In this case permission to photocopy is not required from the publisher.

ISBN 981-02-3009-5

This book is printed on acid-free paper.

Printed in Singapore by Uto-Print 


\section{PREFACE}

In recent years quantum Monte Carlo (QMC), a stochastic method for solving the Schroedinger equation, has become a subject of increased interest in chemistry and physics. As one of the newer approaches in the arsenal of theoretical methods, with only a relatively modest history of development compared to basis set expansion approaches, the method has sprung to the fore because of its capability of yielding computational results of high accuracy.

Formulations of QMC can take on different forms depending on the particular area of application. Emphasis in the present volume is on the electronic structure of atoms and molecules. Also of major interest generally is the application of QMC to the determination of nuclear motion in molecular systems and to statistical mechanics. These latter areas are touched on only briefly in this volume.

The contributions contained herein cover a broad range of topics. In Chapter 1, Dario Bressanini, Pietro Cremaschi, Massimo Mella, and Gabriele Morosi show how information on the exact wave function can be extracted in a QMC simulation and stored in an approximate analytical wave function whose linear and nonlinear parameters are optimized by a least squares criterion. In a related but independent study in Chapter 2, James Anderson, Arne Luechow, and Massimo Mella present a method for direct determination of the difference between the exact wave function and an approximate wave function. They indicate how the difference can be used to correct the trial function to obtain a wave function of higher accuracy.

In Chapter 3, Steven Alexander and Robert Coldwell review a variational Monte Carlo (VMC) method for the computation of atomic energies and present very detailed results for $\mathrm{He}-\mathrm{Ne}$. A hybrid method, one that combines in a novel way both VMC (for nuclear motion) and QMC (for electronic motion), is presented in Chapter 4 by Dario Bressanini and Peter Reynolds and applied to $\mathrm{HD}^{+}$. In Chapter 5 Heinz-Juergen Flad, Michel Caffarel, and Andreas Savin investigate the use of multi-configuration self-consistent field trial wave functions for a class of QMC calculations, fixed node, to ascertain the effect of the addition of various types of configurations on the nodal structure and the energy of the atoms B - F.

In Chapter 6, Kaoru Iguchi summarizes various QMC approaches with representative examples. He extends work on molecules with comments on applications to systems containing a positron. For many molecular studies, 
the most inner-shell electrons play a minor role and can be addressed with the use of effective core potentials. This approach is discussed in Chapter 7 by Carl Greeff, Brian Hammond, and William Lester, Jr. and exemplified by their recent work. In Chapter 8, Lubos Mitras and Jeffrey Grossman also address this approach and present a summary of these recent studies of silicon and carbon systems. They argue persuasively for the promise of QMC for the study of systems containing up to $100-200$ valence electrons with energies consistent with experiment to within a few percent.

The applicability of QMC to positron-containing molecules is the focus in Chapter 9 of David Schrader's presentation. He provides evidence for the thesis that QMC is superior to expansion variational methods for treating positronic systems. Systems of large atomic number present serious sampling problems. In Chapter 10, Stuart Rothstein briefly reviews the various approaches that have been introduced to address this problem including an all-electron partitioning scheme. He also mentions his work with collaborators on the infinitesimal differential diffusion QMC method.

In addition to the use of effective core potentials to eliminate the sampling problem of the innermost core electrons, model potentials have also been introduced for this purpose. In Chapter 11, Takashi Yoshida reviews this area with particular attention to alkaline earth atoms.

Optimization of many-parameter wave functions, which pervades many approaches to VMC and QMC computations, is examined in Chapter 12 by Peter Nightingale and Cyrus Umrigar. These authors review selected applications to atoms, molecules and van der Waals clusters.

I am indebted to the contributing authors for their cooperation in bringing this volume to fruition. I thank Delano Chong, Series Editor, for the opportunity to make this project a reality.

William A Lester, Jr. Berkeley, California November 1996 


\section{CONTENTS}

Preface $\quad v$

1. Analytical Wavefunctions from Quantum Monte Carlo Simulations 1 D. Bressannini, P. Cremaschi, M. Mella, and G. Morosi

2. Quantum Monte Carlo: Direct Determination of the Difference between True and Trial Wavefunctions

J. B. Anderson, A. Luechow, and M. Mella

3. Atomic Calculations Using Variational Monte Carlo

S. A. Alexander and R. L. Coldwell

4. Recent Progress in QMC Simulations of Systems with Multiple Time Scales: Hybrid Nonadiabatic QMC

D. Bressannini and P. J. Reynolds

5. Quantum Monte Carlo Calculations with Multi-Reference Trial Wave Functions

H.-J. Flad, M. Caffarel, and A. Savin

6. Quantum Monte Carlo Calculation of Atoms and Molecules

K. Iguchi

7. Quantum Monte Carlo with Pseudopotentials for Electronic Structure of Atoms and Molecules

C. W. Greeff, W. A. Lester, Jr., and B. L. Hammond

8. Quantum Monte Carlo Study of Si and C Molecular Systems

L. Mitas and J. C. Grossman

9. Positrons: A Challenge and Opportunity for QMC

D. M. Schrader

10. All-Electron Monte Carlo Calculations of Heavy Atom Systems S. M. Rothstein

11. Quantum Monte Carlo Method with Model Potentials

T. Yoshida

12. Monte Carlo Optimization of Trial Wave Functions in Quantum Mechanics and Statistical Mechanics $M$. P. Nightingale and C. J. Umrigar 